\title{
Atomic mechanisms for the formation of charge-density waves in 3-dimensional electronic crystals
}

\author{
Sander van Smaalen ${ }^{1}$, Sitaram Ramakrishnan ${ }^{1}$, Srinivasan Ramakrishnan $^{2}$ \\ ${ }^{1}$ Laboratory of Crystallography, University of Bayreuth, 95447 Bayreuth, Germany, \\ ${ }^{2}$ Department of Condensed Matter Physics and Materials Science, Tata Institute of Fundamental Research, Mumbai 400005, India
}

smash@uni-bayreuth.de

The charge-density wave (CDW) is a modulation of the density of conduction electrons in metals, which is coupled to displacements of the atoms according to a wave with the same wavelength (same wave vector) as the modulation of the conduction band [1]. The classical CDW is the stable state of quasi-1-dimensional (1D) metallic crystals at low temperatures. The latter possess crystal structures with obvious 1D features, like chains of atoms supporting 1D electron bands, and these materials display a highly anisotropic electrical resistance, with the low resistance in the direction of the 1D atomic chains. The mechanism is Fermi-surface nesting (FSN), where a single wave vector $\mathbf{q}$ connects to each other different parts of the Fermi surface. A modulation of the atomic positions according to a wave with this wave vector $\mathbf{q}$ leads to a lowering of the electronic energy. The accompanying elastic strain then leads to an optimal magnitude of the atomic displacements, the latter which can be measured by x-ray diffraction (XRD).

More recently, CDWs have been found in metals whose crystal structures and physical properties lack obvious 1D features [2, 3]. Mechanisms have been put forward, that provide alternative explanations for the formation of CDWs. In particular this includes qdependent electron-phonon coupling (EPC). The EPC peaks at a particular wave vector q. A static modulation with this wave vector then leads to a lowering of the energy of the system, which is not purely electronic, but should be attributed to the EPC terms.

Here, we present comprehensive studies towards CDWs in the materials $\mathrm{La}_{3} \mathrm{Co}_{4} \mathrm{Sn}_{13}, \mathrm{CuV}_{2} \mathrm{~S}_{4}$ and $\mathrm{Er}_{2} \mathrm{Ir}_{3} \mathrm{Si}_{5}$ with strong electron correlations [4-6]. Temperature-dependent transport and thermodynamic properties are correlated with temperature dependent diffraction studies. First-order and second order CDW phase transitions are identified. Crystal structures of the CDW phases are determined, while considering changes of the symmetry along with the development of twinned crystals at the phase transitions. Crystal structures are successfully used to identify the atomic mechanisms of CDW formation and to explain peculiar electronic and magnetic properties. $\mathrm{La}_{3} \mathrm{Co}_{4} \mathrm{Sn}_{13}: P m-3 n$ (No. 223) to $I 2_{1} 3$ (No. 199; 8-fold supercell); $\mathrm{CuV}_{2} \mathrm{~S}_{4}: F d-3 m$ (No. 227) to Imm2( $\sigma 00$ ) (No. 44.1.12.4) and $\mathrm{Er}_{2} \mathrm{Ir}_{3} \mathrm{Si}_{5}: \operatorname{Ibam}$ (No. 72$)$ to $I-1\left(\sigma_{1} \sigma_{2} \sigma_{3}\right)$ (No. 2.1.1.1).

[1] Monceau, P. (2012). Electronic crystals: an experimental overview. Adv. Phys. 61, 325-581. Doi: 10.1080/00018732.2012.719674

[2] Chen, C.-W., Choe, J. \& Morosan, E. (2016). Charge density waves in strongly correlated electron systems. Rep. Prog. Phys. 79, 084505.

[3] Ramakrishnan, S. \& van Smaalen, S. (2017). Unusual ground states in $\mathrm{R}_{5} \mathrm{~T}_{4} \mathrm{X}_{10}(\mathrm{R}=$ rare earth; $\mathrm{T}=\mathrm{Rh}, \mathrm{Ir} ;$ and $\mathrm{X}=\mathrm{Si}, \mathrm{Ge}, \mathrm{Sn})$ : a review. Rep. Prog. Phys. 80, 116501. Doi: 10.1088/1361-6633/aa7d5f.

[4] Ramakrishnan, S., Schönleber, A., Hübschle, C. B., Eisele, C., Schaller, A. M., Rekis, T., Bui, Ng. Hai An, Feulner, F., van Smaalen, S. Bag, B., Ramakrishnan, S., Tolkiehn, M. \& Paulmann, C. (2019). Charge density wave and lock-in transitions of CuV $2 \mathrm{~S}_{4}$. Phys. Rev. B 99, 195140. Doi: 10.1103/PhysRevB.99.195140

[5] Welsch, J., Ramakrishnan, S., Eisele, C., van Well, N., Schönleber, A., van Smaalen, S., Matteppanavar, S., Thamizhavel, A., Tolkiehn, M., Paulmann, C. \& Ramakrishnan, S. (2019). Second order structural and CDW transitions in single crystals of La $\mathrm{Le}_{4} \mathrm{Sn}_{13}$. Phys. Rev. Mater. 3, 125003. Doi: 10.1103/PhysRevMaterials.3.125003

[6] Ramakrishnan, S., Schönleber, A., Rekis, T., van Well, N., Noohinejad, L., van Smaalen, S., Tolkiehn, M., Paulmann, C., Bag, B., Thamizhavel, A., Pal, D. \& Ramakrishnan, S. (2020). Unusual charge density wave transition and absence of magnetic ordering in $\mathrm{Er}_{2} \mathrm{Ir}_{3} \mathrm{Si}_{5}$. Phys. Rev. B 101, 060101(R). Doi: 10.1103/PhysRevB.101.060101

Keywords: Charge density wave; CDW; rare earth compounds; transition metal compounds; sulfides; silicides.

Single-crystal x-ray diffraction data were collected at Beamline P24 of PETRA-III at DESY, Hamburg, Germany and at Beamline BM01 of the SNBL at the ESRF, Grenoble, France. This research has been funded by the Deutsche Forschungsgemeinschaft (DFG, German Research Foundation)_No. 265092781, and the Alexander-von-Humboldt Foundation within their research-group linkage program. 